\title{
Effects of variable resistance on smart structures of cubic reconnaissance satellites in various thermal and frequency shocking conditions ${ }^{\dagger}$
}

\author{
Hassan Elahi ${ }^{1,2}$, Zubair Butt ${ }^{3}$, Marco Eugnei ${ }^{1}$, Paolo Gaudenzi ${ }^{1}$ and Asif Israr $^{2}$ \\ ${ }^{1}$ Department of Mechanical and Aerospace Engineering, La Sapienza University of Rome, Italy \\ ${ }^{2}$ Institute of Space Technology Islamabad, Pakistan \\ ${ }^{3}$ Department of Mechatronics Engineering, University of Engineering \& Technology Taxila, Pakistan
}

(Manuscript Received 000 00, 2016; Revised 000 00, 2017; Accepted 000 00, 2017)

\begin{abstract}
Piezoelectric materials are widely used as smart structures in cubic reconnaissance satellites because of their sensing, actuating, and energy-harvesting abilities. In this study, an analytical model is developed in specific mechanical thermal shocking conditions. A special circuit and apparatus is designed for experimentation on the basis of the inverse piezoelectric effect. An equivalent circuit method is used to establish the relationship between the resistance and peak-to-peak voltage of lead zirconate titanate used as smart materials for cubic reconnaissance satellites. Various frequencies and resistance were applied in different mechanical thermal shocking conditions. Moreover, numerical simulations are conducted in various mechanical loading conditions to determine the accumulative effect. The model provides a novel mechanism to characterize the smart structures in cubic reconnaissance satellites. A rise in temperature increases peak-to-peak voltage; a rise in frequency decreases peak-to-peak voltage; and intensified resistance decreases peak-to-peak voltage. Based on experimentation and simulation, the optimum resistance is predicted for the various frequencies and temperatures. The various conditions may correspond to the different applications of smart structures for cubic reconnaissance satellites. The analytical calculations are in good agreement with experimental and numerical calculations.
\end{abstract}

Keywords: Thermal shocking; Smart structure; Satellites; Frequency shocking; Cubic reconnaissance

\section{Introduction}

Satellites have been used in many military applications, including infrared sensors to track missile launches, electronic sensors to listen to classified conversations, and optical and other sensors to aid in military surveillance. Reconnaissance satellites have also been proven beneficial to civilians, such as with the use of commercially available satellite photographs with surface features and structures (e.g., fire-sensing photographs of remote forested areas). The future of the aerospace industry depends on the development of smart structures (i.e., piezoelectric materials) $[1,32]$. Since the last few decades, the importance of smart structures (i.e., from nanostructures to megastructures) is undeniable. However, to optimize megastructures as microstructures, energy as an external voltage source is needed to sense and actuate a device. To overcome this problem, smart structures (e.g., piezoelectric materials) have been considered because of their self-actuation properties, although their structural dimensions and weight should be optimized for cubic satellites. The future of the aerospace

*Corresponding author. Tel.: +393276544166

E-mail address: hassanelahi_uet@yahoo.com

${ }^{\dagger}$ Recommended by Associate Editor

(C) KSME \& Springer 2017 industry highly depends on the application of these smart structures. On the one hand, smart structures can be directly used as a power source to recharge batteries and provide energy to wireless sensors in satellites; on the other hand, they can be easily molded to any shape [2-4]. Very large space structures are difficult to control because of damping issues, but these problems can be resolved using smart structures [5]. However, a fundamental problem in designing satellites is the effect of resistance and frequency caused by a robust satellite structure [6]. Accordingly, reconnaissance satellite structures can adapt to space environments by utilizing smart materials to dampen the vibrations of active tuned mass dampers [7]. Piezoelectric sensors can also be used as pneumatic controls for different actuators, which in turn can be further utilized in various applications [8]. Furthermore, mechanical thermal shocking conditions are important for space structure stability and workability; accordingly, the Moiré method was proposed because $90 \%$ of the reduction was observed in theodolites [9]. Weight reduction is also important to ensure the robustness and optimization of satellite designs [10]. However, depolarization is complicated at high thermal shocking temperatures near the Curie temperature; at this point modal analysis at various frequency levels can be used to obtain the most opti- 
mized vibration and resistance [11]. An analytical solution for piezoelectric bimorph energy harvesters is presented to observe the range of satellite tuning for various frequency applications and resistances, and ultimately, to achieve a hybrid structure [12]. Subsequently, the electrical and mechanical properties of piezoelectric materials are predicted in different thermal conditions to obtain the optimized results for satellites with smart structures [13]. Energy is wasted when satellites vibrate; however, this energy can be utilized in a relatively good way using piezoelectric patches. Experimental results have verified the importance of piezoelectric patches for the structural stability of satellites [14]. A piezoelectric voltage equation is also developed based on the structure combination theory in which voltage is dependent on frequency [15]. A modal analysis has exhibited how piezoelectric plates can behave differently from piezoelectric beams, in which the results have been predicted by a numerical solution using the Hamilton-Jacobi equation [16, 28-31]. Overall, reconnaissance satellites are widely used for both military and civilian applications because of their spatial resolution, longevity, and surveillance abilities [17, 18].

In previous studies, a cubist satellite with $15 \mathrm{~mm}$ relative displacement showed no change in behavior when its structural change was aimed toward optimization $[19,20]$. In other works, the response of peak-to-peak voltage in various thermal shocking conditions differed from shocking at constant temperature because of degradation [21, 22]. Moreover, dynamic loadings in various frequency and resistance conditions behaved differently for degraded piezoelectric materials, as opposed to static loading in dynamic conditions [23-26].

In this study, a translation model for a cubic reconnaissance satellite with smart structures is developed to predict the response of piezoelectric materials toward thermal shocking in various frequency and resistance conditions. The formulated analytical model is verified by experimentation and simulation. The ultimate goal of this study is to use piezoelectric materials (i.e., smart structure) for the construction of a cubic reconnaissance satellite with a wireless sensor network, as well as for the structural health monitoring of its micro-electro mechanical system.

\section{Analytical modeling of reconnaissance satellites}

Ten piezoelectric patches are mounted on the external surface of the reconnaissance satellites, with two patches specifically placed on one side (Fig. 1(a)). To simplify the analytical model, only one piezoelectric patch is analyzed because the total effect of all patches is the accumulative effect of a single patch (Fig. 1(b)).

The structure comprises a base and a heat filament element for the thermal shocking of the piezoelectric material; both materials are rigid. The mass is connected to a base via a mass spring damper system, while the piezoelectric material is placed between copper electrodes (i.e., cathode and anode), which in turn are connected to the mass and the base. In this

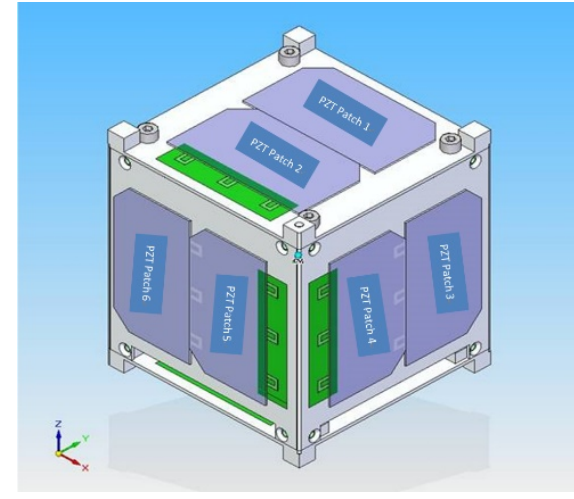

(a)

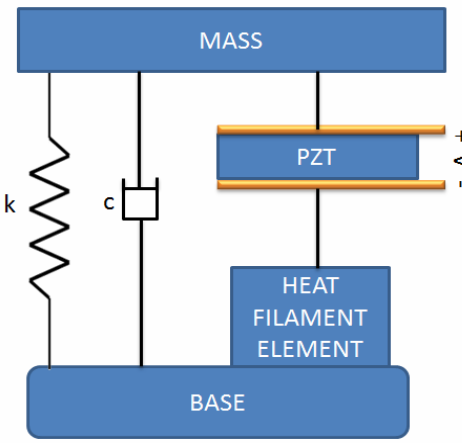

$\uparrow u(t)$

(b)

Fig. 1. (a) Overall structure of reconnaissance satellite; (b) analytical model for single translational piezoelectric patch.

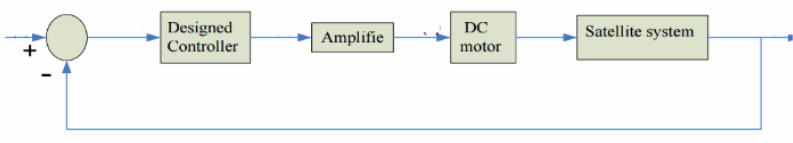

Fig. 2. Closed-loop control system of satellite.

closed-loop system, the transfer function is stabilized by placing all poles in the left part of the root locus. The behavior of the satellite system is represented as a block diagram, as shown in Fig. 2.

In Eq. (1), $M$ is the rigid mass of the base connected to oscillating mass $m$ via damper $c$ and spring $k$. The piezoelectric patch is placed between two electrodes with resistance $R$. The displacement of the oscillating mass is denoted as $u(t)$, displacement of base excitation as $y(t)$, and voltage generated by the piezoelectric patch as $v(t)$. The electromechanical coefficient is represented by $\theta$, while the capacitance of the piezoelectric patch is represented by $C_{p}$. Moreover, $Q_{m}$ is the mechanical quality factor of the piezoelectric material, which is dependent on operating temperature. According to Newton's second law of motion,

$$
M \frac{d^{2} u(t)}{d t^{2}}+c \frac{d u(t)}{d t}+k u(t)-\theta v(t)+Q_{m}(t)=-M \frac{d^{2} y(t)}{d t^{2}},
$$


Table 1. Description of specimen (PZT-5A4E, single-layer patch)

\begin{tabular}{c|c|c|c}
\hline \multirow{2}{*}{ Composition } & \multicolumn{3}{|c}{ Dimension $(\mathrm{cm})$} \\
\cline { 2 - 4 } & Length & Width & Thickness \\
\hline $\begin{array}{c}\text { Lead zirconate } \\
\text { titanate }\end{array}$ & 1.2 & 0.6 & 0.3 \\
\hline
\end{tabular}

Table 2. Parent specimen properties.

\begin{tabular}{|c|c|c|c|c|}
\hline Sr. No. & Description & Notation & Value & Units \\
\hline \multicolumn{5}{|c|}{ Piezoelectric properties } \\
\hline 1 & $\begin{array}{l}\text { Relative dielectric } \\
\text { constant @1 KHz }\end{array}$ & $\mathrm{K}_{3}^{\mathrm{T}}$ & 1,800 & \\
\hline 2 & $\begin{array}{l}\text { Piezoelectric strain } \\
\text { coefficient }\end{array}$ & $\begin{array}{l}d_{33} \\
d_{31}\end{array}$ & $\begin{array}{c}374 \times 10^{-12} \\
-171 \times 10^{-12}\end{array}$ & Meters/volt \\
\hline 3 & $\begin{array}{c}\text { Piezoelectric voltage } \\
\text { coefficient }\end{array}$ & $\begin{array}{l}\mathrm{g}_{33} \\
\mathrm{~g}_{31}\end{array}$ & $\begin{array}{c}24 \times 10^{-3} \\
-11.6 \times 10^{-3}\end{array}$ & $\begin{array}{l}\text { Volt meters/ } \\
\text { Newton }\end{array}$ \\
\hline 4 & Coupling coefficient & $\begin{array}{l}\mathrm{K}_{33} \\
\mathrm{k}_{31} \\
\end{array}$ & $\begin{array}{l}0.72 \\
0.32 \\
\end{array}$ & \\
\hline 5 & Polarization field & $\mathrm{E}_{\mathrm{p}}$ & $2 \times 10^{6}$ & Volts/meter \\
\hline 6 & $\begin{array}{l}\text { Initial depolarization } \\
\text { field }\end{array}$ & $\mathrm{E}_{\mathrm{c}}$ & $5 \times 10^{5}$ & Volts/meter \\
\hline \multicolumn{5}{|c|}{ Mechanical properties } \\
\hline 7 & Density & $\rho$ & 7800 & $\mathrm{Kg} /$ meter $^{3}$ \\
\hline 8 & Elastic modules & $\begin{array}{l}\mathrm{Y}^{\mathrm{E}}{ }_{3} \\
\mathrm{Y}_{1}^{\mathrm{E}}\end{array}$ & $\begin{array}{l}5.2 \times 10^{10} \\
6.6 \times 10^{10} \\
\end{array}$ & $\begin{array}{l}\text { Newton/ } \\
\text { meter }^{2}\end{array}$ \\
\hline \multicolumn{5}{|c|}{ Thermal properties } \\
\hline 9 & $\begin{array}{c}\text { Thermal expansion } \\
\text { coefficient }\end{array}$ & & $\sim 4 \times 10^{-6}$ & $\begin{array}{l}\text { Meters/ } \\
\text { meter }{ }^{\circ} \mathrm{C}\end{array}$ \\
\hline 10 & Curie temperature & & 350 & ${ }^{\circ} \mathrm{C}$ \\
\hline
\end{tabular}

$$
C_{p} \frac{d v(t)}{d t}+\frac{v(t)}{R}+\theta \frac{d u(t)}{d(t)}=0
$$

Resistance, capacitance, and electromechanical coefficients are computed using Eq. (4), where C, L, W and T represent mechanical damping, length, width, and thickness of the piezoelectric patch, respectively.

$$
C=\frac{4 \in_{33} L W}{T}, K=\frac{W T^{3}}{4 L^{3} S_{11}}, \theta=\frac{3 L W d_{31}}{4 T \in_{33}}, Q_{m}=\frac{1}{2 \pi f R C} .
$$

After obtaining the Laplace and conversion of Eqs. (2) and (3) from the time domain to the frequency domain, the simplified equation of the resonance frequency is obtained for voltage generation with base excitation

$$
\frac{v(j w)}{y(j w)}=-\frac{R \theta Q_{m} M w^{2}}{c+j w C R C_{p}+\theta^{2} R} .
$$

\section{Experimentation}

To validate the analytical model, an experimentation was performed for the specifically designed test rig. A rectangular patch of lead zirconate titanate (PZT-5A4E) was selected be-

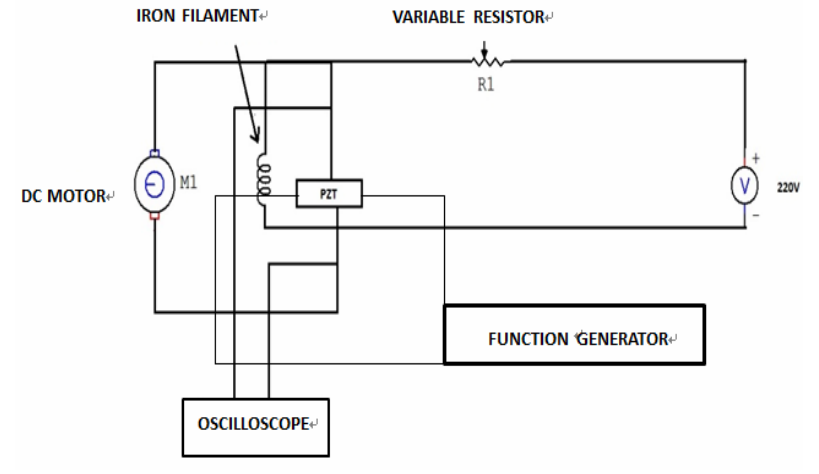

Fig. 3. Experimental setup.

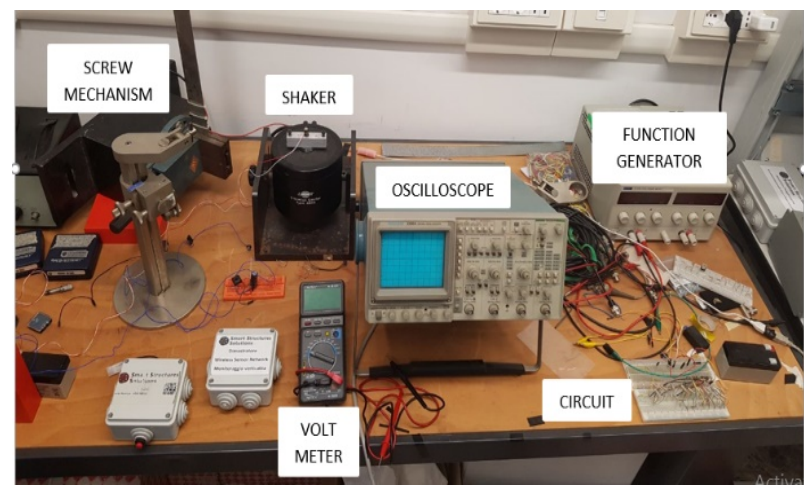

Fig. 4. Circuit diagram of experimental setup.

cause of its excellent dielectric, ferroelectric, and piezoelectric properties. The dimensions and properties of the piezoelectric material are described in Tables 1 and 2, respectively.

The experiment was designed in such a way that the load cell was mounted on the base composite structure, which was used to construct the satellite and to subsequently calculate the force or load on the piezoelectric patch. The piezoelectric patches were placed between the copper electrodes surrounded by a mica sheet to provide thermal and electrical insulation. Temperatures at any given instance or spot were analyzed by a thermal shocking heat filament and a laser temperature gun. To electrically shock the piezoelectric material at various frequencies and resistances, a function generator and a decade box were utilized. For the mechanical loading, a DC motor with a screw mechanism was used to control the amount of force acting on the structure. To analyze the overall result, a digital oscilloscope was utilized with a data acquisition system. The results of the experimentation validated the results of the analytical model. The experimental setup is shown in Fig. 3 and the circuit diagram is shown in Fig. 4.

A mechanical stimulus was applied alternately to the PZT $[21,27]$. The circuit was designed to shift the applied stress via a DC motor with a specified RPM setting and shaft geometry (Fig. 5). Twenty-three sets of experimentation were performed for each dataset. After each experimentation, the PZT-5A4E patches were "wasted" to achieve the effect of polarization. A total of 1472 patch experimentations were 
Table 3. Material properties for simulation.

\begin{tabular}{c|c}
\hline Mechanical properties & PZT-5A4E \\
\hline Elastic coefficient $(\mathrm{GPa})$ & 121 \\
C11 & 75.40 \\
$\mathrm{C} 12$ & 75.20 \\
$\mathrm{C} 13$ & 111 \\
$\mathrm{C} 33$ & 21.1 \\
$\mathrm{C} 44$ & \\
Piezoelectric coupling matrix & \\
$\left(10^{\wedge}-12 \mathrm{~m} / \mathrm{v}\right)$ & 584 \\
$\mathrm{~d} 15$ & -171 \\
$\mathrm{~d} 31$ & 374 \\
$\mathrm{~d} 33$ & \\
$\mathrm{e} 31$ & -5.4 \\
$\mathrm{e} 33$ & 15.8 \\
$\mathrm{e} 15$ & 12.3 \\
\hline Piezoelectric coefficient $(\mathrm{C} / \mathrm{m} 2)$ & \\
\hline Dielectric constant $(10-9 \mathrm{~F} / \mathrm{m})$ & 8.107 \\
$\in 11$ & 7.346 \\
\hline
\end{tabular}

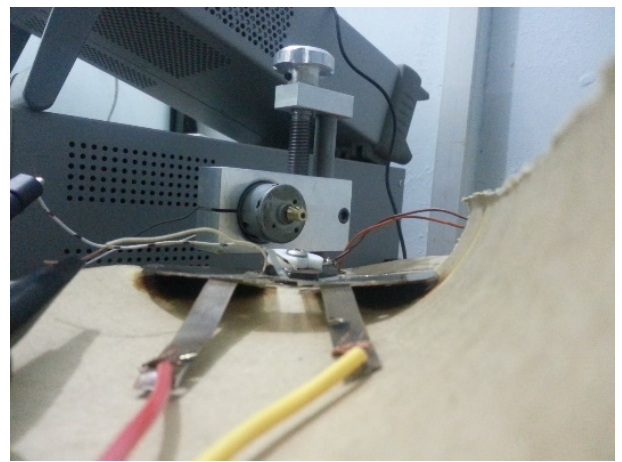

Fig. 5. Experimental setup of alternate mechanical stress application.

performed in variable mechanical-electrical shocking and thermal conditions.

\section{Simulation}

The analytical and experimental results validated the numerical simulation method. Simulation was conducted in ABAQUSTM by considering a finite element 3D brick model of PZT-5A4E; its dimensions are listed in Table 1. The material properties required for simulation are given in Tables 2 and 3 .

In ABAQUS, the Newton integration technique was used for the simulation. Eight hexahedral C3D8E nodal firstcoupled field elements were also developed. The final meshed model of the PZT-5A4E contained 8000 hexahedral elements and 9741 nodes. Simulations were conducted according to the conditions generated during experimentation and dynamic analysis. The core material of the beam was modeled with C3D20 elements, while all piezoelectric strips were modeled using C3D20E elements. A perfect bond between the core and piezoelectric materials was defined with a surface-based tie constraint in which the piezoelectric surfaces were retained as

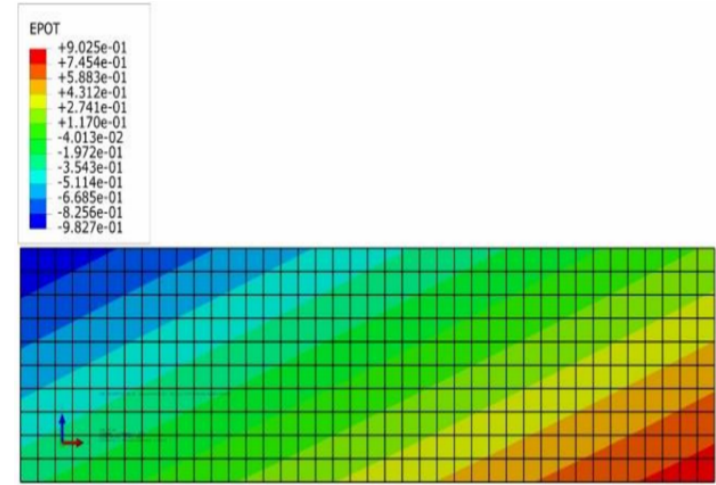

Fig. 6. Nodal simulation of PZT-5A4E in ABAQUS

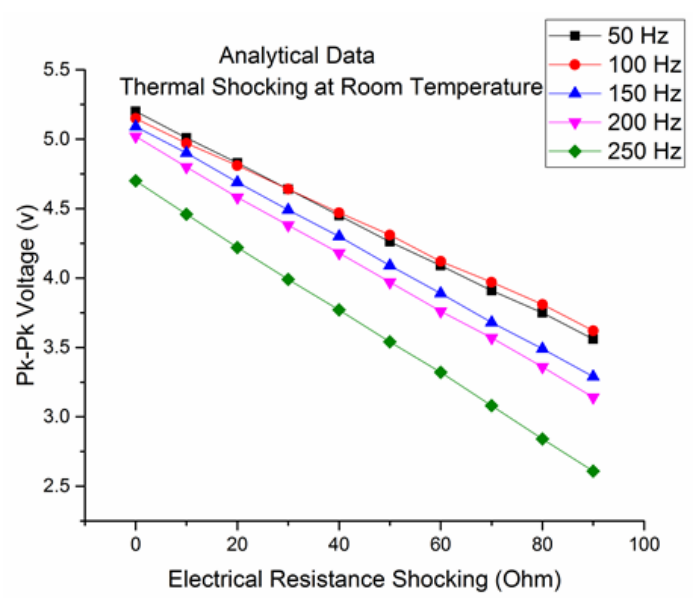

Fig. 7. Analytical data of electrical resistance shocking versus $\mathrm{Pk}-\mathrm{Pk}$ voltage in various frequency shocking conditions at room temperature.

master surfaces. Based on linear constraint equations, the electrical potentials of the top and the bottom surfaces of each piezoelectric strip were coupled to the electrical potentials of the master nodes, which were assigned to each surface. Moreover, the electrical potentials and reaction charges were monitored at the master nodes. The piezoelectric surfaces that bonded with the core material were assigned a zero electrical potential in the analysis. The nodal simulation of PZT-5A4E is shown in Fig. 6.

\section{Results and discussion}

During experimentation, a rise in temperature resulted in an increase in peak-to-peak voltage. By contrast, an increase in frequency resulted in a decrease in output peak-to-peak voltage. For maximum results, optimization was conducted by increasing temperature, decreasing resistance, and decreasing frequency. Subsequent experimentations were performed at the $20-180{ }^{\circ} \mathrm{C}$ temperature range, $0-90 \mathrm{Ohm}$ resistance range, and $50-250 \mathrm{~Hz}$ frequency range. The results are shown in Figs. 7-11. The maximum error for the analytical model, numerical model, and experimental data was about $9.8 \%$.

At about $40 \mathrm{Ohm}$ resistance (Fig. 11), the Pk-Pk voltage 


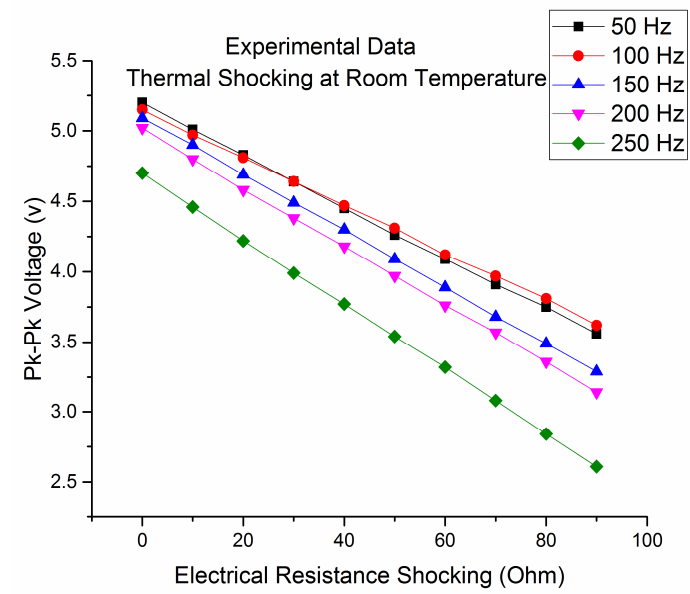

Fig. 8. Experimental data of electrical resistance shocking versus Pk$\mathrm{Pk}$ voltage in various frequency shocking conditions at room temperature.

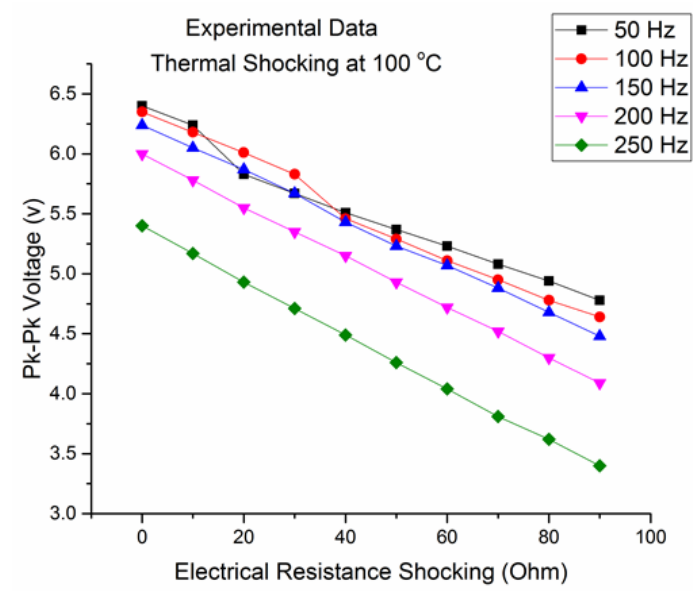

Fig. 9. Experimental data of electrical resistance shocking versus Pk$\mathrm{Pk}$ voltage in various frequency shocking conditions at $100{ }^{\circ} \mathrm{C}$ thermal shocking.

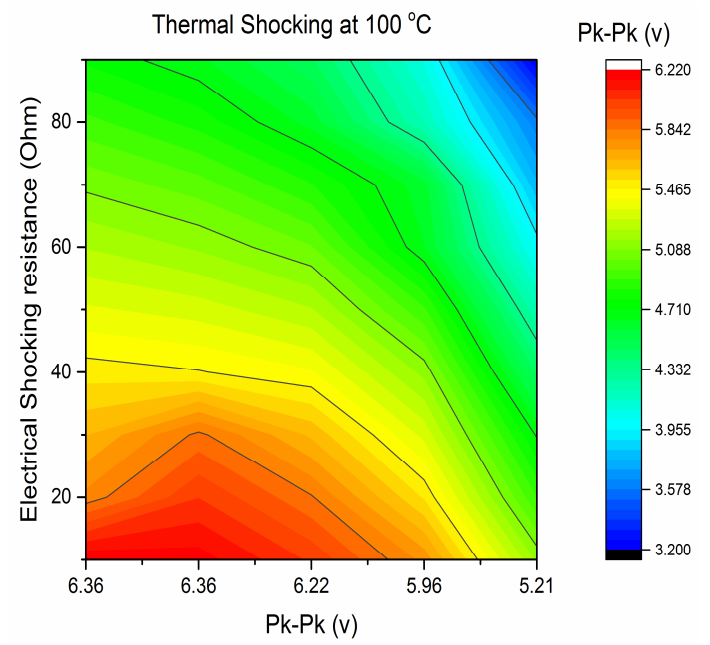

Fig. 10. Numerical simulation of electrical resistance shocking versus $\mathrm{Pk}-\mathrm{Pk}$ voltage in various frequency shocking conditions at $100{ }^{\circ} \mathrm{C}$ thermal shocking.

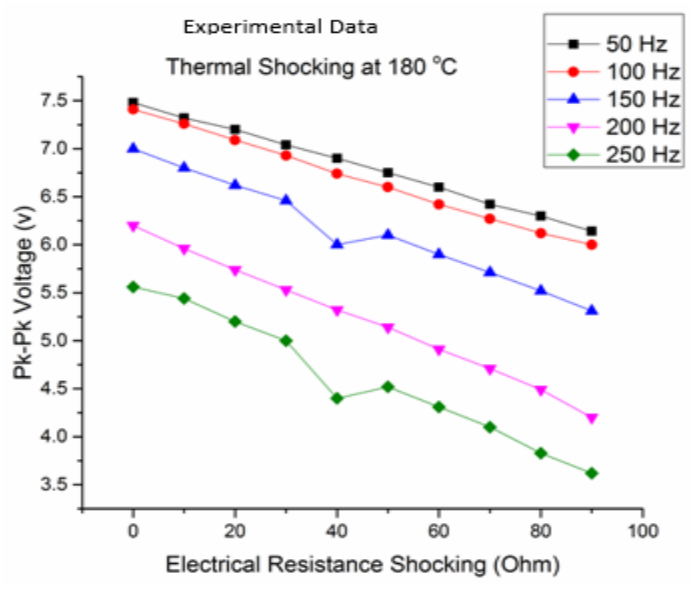

Fig. 11. Experimental data of electrical resistance shocking versus Pk$\mathrm{Pk}$ voltage in various frequency shocking conditions at $180^{\circ} \mathrm{C}$ thermal shocking.

dropped abruptly; at this point, the effect of polarization decreased, thereby resulting in the reduced doping of ions.

\section{Conclusions}

In this study, an analytical model with a single degree of freedom was developed and then verified by numerical and experimental results. The following conclusions are presented based on the analytical, experimental, and numerical solutions:

(1) By increasing the temperature to Curie temperature, the peak-to-peak voltage of the PZT-5A4E increased as a linear function. By contrast, increasing the frequency linearly decreased the peak-to-peak voltage.

(2) A negative linear behavior was observed between frequency and peak-to-peak voltage, as well as for resistance and peak-to-peak voltage.

(3) Resistance was an important factor for the piezoelectric materials, specifically when solving for resonant and antiresonant frequencies.

(4) At high resistance and frequency, the motion of ions was restricted. Subsequently, the rate of polarization decreased, which resulted in a low peak-to-peak voltage. Therefore, PZT5A4E is highly applicable in high-temperature, low-resistance, and low-frequency conditions.

(5) A sudden drop in Pk-Pk voltage was observed at approximately $40 \mathrm{Ohm}$ resistance; thus, using PZT in this condition should be avoided.

(6) For best performance and maximum $\mathrm{Pk}-\mathrm{Pk}$ voltage, PZT can be used at $180{ }^{\circ} \mathrm{C}$ (temperature), $20 \mathrm{Ohm}$ (resistance), and $0 \mathrm{~Hz}$ (frequency). In this specific condition, ionic dipolar motion is at its maximum, thereby preventing resonating frequencies.

\section{Nomenclature}

$\mathrm{t} \quad$ : Time

$\mathrm{v}(\mathrm{t}) \quad$ : Voltage 
$\mathrm{u}(\mathrm{t}) \quad$ : Displacement of mass

$y(t) \quad$ : Displacement due to base excitation

u(jw) : Displacement in frequency domain

$y(j w)$ : Displacement due to base excitation in frequency domain

w : Angular frequency of harvester

$\theta \quad$ : Electromechanical coupling coefficient

$\mathrm{K} \quad$ : Mechanical stiffness of material

$\mathrm{R} \quad$ : Resistance

C : Mechanical damping

$\mathrm{C}_{\mathrm{p}} \quad$ : Capacitance of piezoelectric material

$\mathrm{Q}_{\mathrm{m}} \quad$ : Mechanical quality factor

\section{References}

[1] A. K. Noor et al., Structures technology for future aerospace systems, Computers \& Structures, 74 (5) (2000) 507-519.

[2] V. G. M. Annamdas and C. K. Soh, Contactless load monitoring in near-field with surface localized spoof plasmonsA new breed of metamaterials for health of engineering structures, Sensors and Actuators A: Physical, 244 (2016) 156-165.

[3] Q. Lei et al., Design and experiment of PZT network-based structural health monitoring scanning system, Chinese Journal of Aeronautics, 22 (5) (2009) 505-512.

[4] D. Vasic, Y.-Y. Chen and F. Costa, Self-powered piezoelectric energy harvester for bicycle, Journal of Mechanical Science and Technology, 28 (7) (2014) 2501-2510.

[5] P. Gasbarri, R. Monti and M. Sabatini, Very large space structures: Non-linear control and robustness to structural uncertainties, Acta Astronautica, 93 (2014) 252-265.

[6] P. Gasbarri et al., Effects of uncertainties and flexible dynamic contributions on the control of a spacecraft fullcoupled model, Acta Astronautica, 94 (1) (2014) 515-526.

[7] N. R. Fisco and H. Adeli, Smart structures: Part I-Active and semi-active control, Scientia Iranica, 18 (3) (2011) 275284.

[8] M. Kamali et al., Integrated nozzle - flapper valve with piezoelectric actuator and isothermal chamber: A feedback linearization multi control device, Journal of Mechanical Science and Technology, 30 (5) (2016) 2293-2301.

[9] D. Zhengchun et al., Design and application of composite platform with extreme low thermal deformation for satellite, Composite Structures (2016).

[10] J. C. Williams and E. A. Starke, Progress in structural materials for aerospace systems11The Golden Jubilee IssueSelected topics in Materials Science and Engineering: Past, Present and Future, edited by S. Suresh, Acta Materialia, 51 (19) (2003) 5775-5799.

[11] J. Fialka et al., Measurement of thermal depolarization effects in piezoelectric coefficients of soft PZT ceramics via the frequency and direct methods, Journal of the European Ceramic Society, 36 (11) (2016) 2727-2738.

[12] H. Madinei et al., Design of MEMS piezoelectric harvesters with electrostatically adjustable resonance frequency,
Mechanical Systems and Signal Processing.

[13] S. Azizi et al., A conceptual study on the dynamics of a piezoelectric MEMS (Micro Electro Mechanical System) energy harvester, Energy, 96 (2016) 495-506.

[14] H.-H. Huang and K.-S. Chen, Design, analysis, and experimental studies of a novel PVDF-based piezoelectric energy harvester with beating mechanisms, Sensors and Actuators A: Physical, 238 (2016) 317-328.

[15] S.-C. Huang and C.-Y. Tsai, Theoretical analysis of a new adjustable broadband PZT beam vibration energy harvester, International Journal of Mechanical Sciences, 105 (2016) 304-314.

[16] S. Porn et al., Level set based structural optimization of distributed piezoelectric modal sensors for plate structures, International Journal of Solids and Structures, 80 (2016) 348-358.

[17] A. S. Belward and J. O. Skøien, Who launched what, when and why; trends in global land-cover observation capacity from civilian earth observation satellites, ISPRS Journal of Photogrammetry and Remote Sensing, 103 (2015) 115-128.

[18] D. P. Roy et al., Landsat-8: Science and product vision for terrestrial global change research, Remote Sensing of Environment, 145 (2014) 154-172.

[19] L. Olivieri and A. Francesconi, Design and test of a semiandrogynous docking mechanism for small satellites, Acta Astronautica, 122 (2016) 219-230.

[20] N. Bérend and X. Olive, Bi-objective optimization of a multiple-target active debris removal mission, Acta Astronautica, 122 (2016) 324-335.

[21] H. Elahi, R. Pasha and M. Khan, Experimental determination of mechanical quality factor of lead zirconate titanate (PZT-5A4E) by equivalent circuit method under various thermal and resistance conditions, University of Engineering and Technology Taxila, Technical Journal, 19 (2) (2014) 1.

[22] P. Liu et al., Transient thermal shock fracture analysis of functionally graded piezoelectric materials by the extended finite element method, International Journal of Solids and Structures, 51 (11-12) (2014) 2167-2182.

[23] J. Boughaleb et al., Design and performance benchmark of various architectures of a piezoelectric bimetallic strip heat engine, Optical Materials, 56 (2016) 110-115.

[24] G. G. Sheng and X. Wang, Response and control of functionally graded laminated piezoelectric shells under thermal shock and moving loadings, Composite Structures, 93 (1) (2010) 132-141.

[25] S. S. Vel and B. P. Baillargeon, Analysis of static deformation, vibration and active damping of cylindrical composite shells with piezoelectric shear actuators, Journal of Vibration and Acoustics, 127 (4) (2005) 395.

[26] J. Sladek et al., Fracture analysis in piezoelectric semiconductors under a thermal load, Engineering Fracture Mechanics, 126 (2014) 27-39.

[27] Z. Butt, R. A. Pasha, F. Qayyum, Z. Anjum N. Ahmad and H. Elahi, Generation of electrical energy using lead zirconate titanate (PZT-5A) piezoelectric material: analytical, numeri- 
cal and experimental verifications, Journal of Mechanical Science and Technology, 30 (2016) 3553-3558.

[28] H. Elahi et al., Robust vehicle suspension system by converting active \& passive control of a vehicle to semi-active control system analytically, Journal of Automation and Control Engineering, 4 (4) (2016).

[29] S. Waqar et al., Effect of drilling parameters on hole quality of Ti-6Al-4V titanium alloy in dry drilling, Materials Science Forum. 2017. Trans Tech Publications.

[30] S. Riaz et al., Vibration feature extraction and analysis for fault diagnosis of rotating machinery-A literature survey, Asia Pacific Journal of Multidisciplinary Research, 5 (1) (2017).

[31] M. A. Gull, H. Elahi, M. Marwat and S. Waqar, A new approach to classification of upper limb and wrist movements using EEG signals, Biomedical Engineering (BioMed), 2017 13th IASTED International Conference on, IEEE (2017) 181-194.

[32] P. Gaudenzi, Smart structures: Physical behaviour, mathematical modelling and applications, John Wiley \& Sons (2009). 